\title{
What do alexithymia items measure? A discriminant content validity study of the Toronto-Alexithymia-Scale $\mathbf{- 2 0}$
}

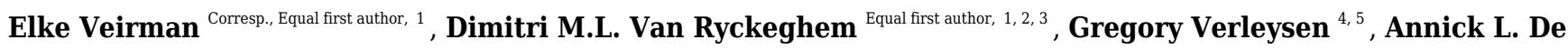 \\ Paepe $^{1}$, Geert Crombez ${ }^{1}$ \\ 1 Department of Experimental-Clinical and Health Psychology, Faculty of Psychology and Educational Sciences, Ghent University, Ghent, Belgium \\ Institute for Health and Behaviour, INSIDE, University of Luxembourg, Esch-sur-Alzette, Luxembourg \\ 3 Experimental Health Psychology, Faculty of Psychology and Neuroscience, Maastricht University, Maastricht, Netherlands \\ 4 Centre for Research and Innovation in Care, Faculty of Medicine and Health Sciences, University of Antwerp, Antwerp, Belgium \\ 5 End-of-Life Care research group, Faculty of Medicine and Pharmacy, Vrije Universiteit Brussel, Brussels, Belgium \\ Corresponding Author: Elke Veirman \\ Email address: elke.veirman@ugent.be
}

Background. Questions have been raised about whether items of alexithymia scales assess the construct alexithymia and its key features, and no other related constructs. This study assessed the (discriminant) content validity of the most widely used alexithymia scale, i.e., the Toronto Alexithymia Scale (TAS-20).

Methods. Participants $(n=81)$ rated to what extent TAS-20 items and items of related constructs were relevant for assessing the constructs 'alexithymia', 'difficulty identifying feelings', 'difficulty describing feelings', 'externally-oriented thinking', 'limited imaginal capacity', 'anxiety', 'depression', and 'health anxiety'.

Results. Results revealed that, overall, the TAS-20 did only partly measure 'alexithymia'. Only the subscales 'difficulty identifying feelings' and 'difficulty describing feelings' represented 'alexithymia' and their intended construct, although some content overlap between these subscales was found. In addition, some items assessed (health) anxiety equally well or even better.

Conclusions. Revision of the TAS-20 is recommended to adequately assess all key features of alexithymia. Findings with the TAS-20 need to be interpreted with caution in people suffering from medical conditions. 


\section{What do Alexithymia Items Measure? A Discriminant 2 Content Validity Study of the Toronto-Alexithymia- 3 Scale - 20}

Elke Veirman $^{1 *}$, Dimitri M.L. Van Ryckeghem ${ }^{1,2,3}{ }^{*}$, Gregory Verleysen ${ }^{4,5}$, Annick L. De Paepe $^{1}$, Geert Crombez ${ }^{1}$

${ }^{1}$ Department of Experimental-Clinical and Health Psychology, Faculty of Psychology and Educational Sciences, Ghent University, Ghent, Belgium

${ }^{2}$ Institute for Health and Behaviour, INSIDE, University of Luxembourg, Esch-sur-Alzette, Luxembourg

${ }^{3}$ Experimental Health Psychology, Faculty of Psychology and Neuroscience, Maastricht University, Maastricht, Netherlands

${ }^{4}$ End-of-Life Care research group, Faculty of Medicine and Pharmacy, Vrije Universiteit Brussel, Brussels, Belgium

${ }^{5}$ Centre for Research and Innovation in Care, Faculty of Medicine and Health Sciences, University of Antwerp, Antwerp, Belgium

* The first two authors contributed equally.

Corresponding Author:

Elke Veirman ${ }^{1}$

Henri-Dunantlaan 2, Ghent, 9000, Belgium

Email address: elke.veirman@ugent.be

\section{Abstract}

Background. Questions have been raised about whether items of alexithymia scales assess the construct alexithymia and its key features, and no other related constructs. This study assessed the (discriminant) content validity of the most widely used alexithymia scale, i.e., the Toronto Alexithymia Scale (TAS-20).

Methods. Participants $(\mathrm{n}=81)$ rated to what extent TAS-20 items and items of related constructs were relevant for assessing the constructs 'alexithymia', 'difficulty identifying feelings', 'difficulty describing feelings', 'externally-oriented thinking', 'limited imaginal capacity', 'anxiety', 'depression', and 'health anxiety'.

Results. Results revealed that, overall, the TAS-20 did only partly measure 'alexithymia'. Only the subscales 'difficulty identifying feelings' and 'difficulty describing feelings' represented 'alexithymia' and their intended construct, although some content overlap between these subscales was found. In addition, some items assessed (health) anxiety equally well or even better. 
39 Conclusions. Revision of the TAS-20 is recommended to adequately assess all key features of

40 alexithymia. Findings with the TAS-20 need to be interpreted with caution in people suffering

41 from medical conditions.

\section{Introduction}

The alexithymia construct has been introduced in the early seventies by Sifneos $(1972,1973)$ to describe clinical observations of patients with classic psychosomatic diseases who had difficulty engaging in insight-oriented psychotherapy (e.g., MacLean, 1949; Marty \& de M'Uzan, 1963; Ruesch, 1948; Sifneos, 1967). Since then alexithymia, defined as the inability to recognize and express emotions (Taylor, Bagby, \& Parker, 2016), has been considered a key construct in many theoretical models of health psychology (Lumley et al., 2007). Contemporary theories describe alexithymia as a multidimensional construct with four interrelated features: "(1) difficulty identifying feelings and distinguishing between feelings and the bodily sensations of emotional arousal, (2) difficulty describing feelings to other people, (3) constricted imaginal processes, as evidenced by a paucity of fantasies, and (4) a stimulus-bound, externally oriented cognitive style" (Taylor et al., 1997, p. 29; see also Sifneos, 1994). Over the last decades, alexithymia has been recognized as a risk factor for various psychiatric and medical conditions (Corcos \& Speranza, 2003; Taylor et al., 1997). Particularly, it has been theorized that alexithymia reflects a deficit in the cognitive processing and regulation of emotions (Taylor, 1994; Taylor et al., 1997). This deficit would increase one`s vulnerability for psychiatric and medical conditions (Fernandez, Jazaieri, \& Gross, 2016). This idea is furthermore supported by abundant research showing that levels of alexithymia are increased in patients suffering from illnesses, such as eating disorders (e.g., Taylor, Parker, Bagby, \& Bourke, 1996), posttraumatic stress disorders (e.g., Frewen, Pain, Dozois, \& Lanius, 2006), chronic pain (e.g., Pecukonis, 2009), cancer (e.g., Todarello, La Pesa, Zaka, Martino, \& Lattanzio 1989), and many more (Luminet, Bagby, \& Taylor, 2018; for a review, see Taylor \& Bagby, 2000; Taylor, 2004).

The Toronto Alexithymia Scale - 20 (TAS-20; Bagby, Parker, \& Taylor, 1994a; Bagby, Taylor, \& Parker, 1994b) is worldwide the most frequently used measure of alexithymia in both research and clinical practice (Lane et al., 2015; Sekely, Bagby \& Porcelli, 2018). Although the TAS-20 is considered to be a well validated self-report measure of alexithymia (e.g., Bagby, Taylor, \& Parker, 2020), some concerns about its validity remain (Bermond, Oosterveld, \& Vorst, 2015; Lane et al., 2015; Lumely, Neely, \& Burger, 2007). First, doubts have been raised about whether the TAS-20 measures alexithymia in a comprehensive and relevant manner. The TAS-20 contains three subscales, i.e., 'difficulty identifying feelings', 'difficulty describing feelings', and 'externally-oriented thinking'. The items for assessing the daydreaming factor in the earlier revision of the original TAS (TAS-R; Taylor, Ryan, \& Bagby, 1985; Taylor, Bagby, $\&$ Parker, 1992), were eliminated because of either low item-total correlations or high correlations with a social desirability measure (Bagby et al., 1994a, 1994b). Bagby and colleagues (1994a, 1994b, see also Taylor et al., 2016) motivated their decision by arguing that 
79 Bagby, 2013; Bagby et al., 2020). Furthermore, confirmatory and exploratory factor analyses 80 show that at least half of the externally-oriented thinking items load poorly on their intended 81 factor (factor loadings < .40; e.g., Kooiman et al., 2002; Preece et al., 2017; Taylor, Bagby, \& 82 Parker, 2003). If aspects of the construct alexithymia are underrepresented by the TAS-20 items and/or TAS-20 items are not relevant for the construct, it reflects a lack of content validity.

Second, there are doubts about whether the TAS-20 is sufficiently distinct from measures assessing related theoretical constructs. Some authors have argued that the TAS-20 is a measure of psychological distress rather than alexithymia (Leising, Grande, \& Faber, 2009). Indeed, significant and substantial correlations have been reported between the TAS-20 and measures of anxiety and depression in clinical samples (e.g., Marchesi, Ossola, Tonna, \& De Panfilis, 2014) and in the general population (e.g., Honkalampi et al., 2010). Furthermore, Shahidi and colleagues (2012) found significant correlations between the TAS-20 scores and a measure of health anxiety. This study revealed that the 'difficulty identifying feelings' subscale predicted $52 \%$ of the total variance in health anxiety scores, and argued that this strong relationship is driven by particular items that measure difficulty in differentiating between bodily feelings and emotions (see also Barsky, 2001; De Gucht, Fischler, \& Heiser, 2004; Nakao et al., 2002). It is key that correlations between the TAS-20 and health anxiety are not (partially) explained by content overlap. If the TAS-20 is contaminated by content relevant to related constructs such as anxiety, depression, and health anxiety, relationships between the measures of these constructs may then simply be due to content overlap resulting in inflated explanatory power of alexithymia and hazardous theory building (Dixon \& Johnston, 2019).

Despite these concerns, no study has examined the content validity and discriminant content validity of the TAS-20. In the current study, TAS-20 items are evaluated using the Discriminant Content Validity method (DCV; Johnston et al., 2014), a systematic and transparent way of investigating and reporting whether items are relevant for measuring target theoretical constructs (a key feature of content validity) and whether items are distinct from the content from other theoretical constructs (discriminant content validity). More specifically, we investigated to what extent items from the TAS-20 are (a) relevant for the construct 'alexithymia', and its key features, i.e., 'difficulty identifying feelings', 'difficulty describing feelings', 'externally-oriented thinking', and 'limited imaginal capacity' (content validity), and (b) distinct from related constructs, i.e., 'anxiety', 'depression', and 'health anxiety'

110 (discriminant content validity).

111

\section{Materials \& Methods}

\section{Participants}

114 Participants were 81 psychology students (English track) recruited at Maastricht

115 University via Sona Systems, a cloud-based participant pool management software package

116 (https://maastricht-fpn.sona-systems.com). They were not experts in alexithymia research as we

117 wanted people to decide whether an item assesses a construct based upon the item and construct

118 definition without knowledge bias (i.e., a theoretical background in the field of alexithymia). 
119 Data from participants were only included for the statistical analysis when participants were able

120 to complete the online assessment in line with given instructions and quality checks

121 (performance criteria).

\section{Discriminant content validity method}

123 The Discriminant Content Validity method (DCV) method is a quantitative procedure to

124 assess the (discriminant) content of theory-based measures (for a detailed overview of the

125 methodology, see Johnson et al., 2014). Here, we describe the DCV questionnaire we developed

126 in five steps:

127 Step 1: Identification of constructs

128

129

Eight constructs were identified to be used for the categorization of the items. These constructs were 'alexithymia', 'difficulty identifying feelings', 'difficulty describing feeling', 'externally-oriented thinking', 'limited imaginal capacity', 'anxiety', 'depression', and 'health anxiety'. The constructs 'alexithymia', 'difficulty identifying feelings', 'difficulty describing feelings', 'externally-oriented thinking', and 'limited imaginal capacity' were selected to investigate to what extent TAS-20 items are identified as items that assess alexithymia, and to what extent they are identified to assess the respective key features of alexithymia (content validity). The categories 'anxiety', 'depression', and 'health anxiety' were selected to investigate to what extent the TAS-20 item-content could be differentiated from other constructs to which alexithymia has been related (discriminant content validity). Finally, an 'other' category was added, preventing the impression that all items had to be categorized as measures of one of the predefined constructs.

Step 2: Construct definitions

Definitions were formulated for each of the identified constructs. The definition of alexithymia was based upon the definition of alexithymia provided by the online Oxford Living Dictionaries for English (https://en.oxforddictionaries.com accessed on 11/10/2018). This definition is a representation of how the construct is understood in lay terms and also corresponds to the scientific definition that is widely accepted (Taylor et al., 2016). For the alexithymia features, definitions were based upon the widely acknowledged definitions of Taylor and colleagues (1997). For the other predefined constructs, there are multiple definitions available, which could introduce bias in our findings due to preferring the definition of one theoretical framework over another. Therefore, we opted to base our definitions on those provided by the Online Oxford Living Dictionaries for English (https://en.oxforddictionaries.com accessed on 11/10/2018). The following definitions were used: (1) alexithymia: 'The inability to recognize one's own emotions and to express them, especially in words'; (2) difficulty identifying feelings: 'Difficulty identifying feelings and distinguishing between feelings and the bodily sensations of emotional arousal.'; (3) difficulty describing feelings: 'Difficulty describing feelings to other people. '; (4) externally-oriented thinking: 'A stimulus-bound, externally oriented cognitive style. '; (5) limited imaginal capacity: 'Constricted imaginal processes, as evidenced by a paucity of fantasies.'; (6) anxiety: 'A feeling of worry, nervousness, or unease about something with an uncertain outcome.'; (7) depression: 'Feelings of severe despondency and dejection.'; and (8) 
159 health anxiety: 'A feeling of worry, nervousness, or unease about one's health.'

160

161

162

163

164

165

166

167

168

169

170

171

172

173

174

175

176

177

178

179

180

181

182

183

184

185

186

187

188

189

190

191

192

193

194

195

196

197

198

\section{Step 3: Selection of alexithymia items}

The TAS-20 comprises 20 items across three subscales, with most of the items positively keyed (+) and some negatively keyed (-): 'difficulty identifying feelings' (items $1+, 3+, 6+, 7+$, 9+, 13+, and 14+; e.g., "I am often confused about what emotion I am feeling"), 'difficulty describing feelings' (items $2+, 4-, 11+, 12+$, and 17+; e.g., "It is difficult for me to find the right words for my feelings"), and 'externally-oriented thinking' (items 5-, 8+, 10-, 15+, 16+, 18-, 19-, and 20+; e.g., "I prefer to analyze problems rather than just describe them"). Items are displayed in supplementary information (Table 1 in S1 file).

\section{Step 4: Selection of items for the other constructs}

For 'anxiety', four items (e.g., "I felt fearful") were retrieved from the PROMIS ${ }^{\circledR}$ Item Bank v1.0-Emotional Distress-Anxiety - Short Form 4a (PROMIS-A; Pilkonis et al., 2011; Table 2 in S1 file). For 'depression', four items (e.g., "I felt hopeless") were retrieved from the PROMIS ${ }^{\circledR}$ Item Bank v1.0 - Emotional Distress-Depression - Short Form 4a (PROMIS-D; Pilkonis et al., 2011; Table 3 in S1 file). For 'health anxiety', four items (e.g., "I usually think that I am seriously ill") were retrieved from the Short Health Anxiety Inventory (SHAI; Salkovskis, Rimes, Warwick, \& Clark, 2002; Table 4 in S1 file). For feasibility reasons (i.e., reducing fatigue effects), the number of items for each contrast construct was limited to four.

\section{Step 5: Rating scale of items}

Participants were instructed to rate two questions per construct for each item (e.g., Johnston et al., 2014). In the first question, participants were asked to judge whether an item assesses a particular construct (common-scored items: 'no' and 'yes when reverse scored' $=-1$, whereas 'yes' $=1$; reverse-scored items: 'no' and 'yes' $=-1$, whereas 'yes when reverse scored' $=1$ ). In the second question, participants were asked to indicate on an 11 point scale $(0=0 \%$ confidence to $10=100 \%$ confidence) to what extent they were confident about their judgment. Weighted judgements were calculated to express the relationship between each item and each construct. The code of the answer for 'no', 'yes', and 'yes when reverse scored' was multiplied with its accompanied confidence score, resulting in an outcome score with values ranging from -10 to +10 .

\section{Self-report measures}

\section{Participant characteristics}

After completion of the DCV items, participants were asked to provide demographic information including gender, age, nationality, ethnicity, and current health status.

\section{PROMIS Health Profile}

To provide information on the physical and mental health of the participants, the PROMIS ${ }^{\circledR}$ Profile - v2.1 - PROMIS-29 was filled out, which contains seven scales, i.e., physical function (4 items), anxiety (4 items), depression (4 items), fatigue (4 items), sleep disturbance (4 items), ability to participate in social roles and activities (4 items), pain interference (4 items), and a pain intensity item. All items, except for the pain intensity item, are scored on a 5-point Likert scale. The pain intensity item "In the last 7 days, how would you rate your pain on average?" is rated on a 11-point Likert scale ranging from 0 (no pain) to 10 (worst imaginable 
199

200

201

202

203

204

205

206

207

208

209

210

211

212

213

214

215

216

217

218

219

220

221

222

223

224

225

226

227

228

229

230

231

232

233

234

235

236

237

238

pain) (Hays et al., 1994). Scale summary scores are transformed into a standardized T-scores with a mean of 50 and a standard deviation (SD) of 10. Higher scores reflect more of the concept being measured. Research indicated that this questionnaire is reliable and valid for assessing health-related quality of life in the general population and in populations with chronic health conditions (Hays et al., 2018; Rose et al., 2019).

\section{Detection of careless responding}

Detection of careless responding (e.g., Meade \& Craig, 2012; Oppenheimer, Meyvis, \& Davidenko, 2009) was built-in via three ways. First, the 'other' category provided the opportunity to check whether participants followed the given instructions. In particular, it was considered impossible for participants to provide the same extreme scores (i.e., -10 or +10 ) for an item on all predefined constructs and the 'other' category. Second, the DCV items were intermixed with three items from the Instructional Manipulation Check (IMC; e.g., "Please check yes and 30\% for all constructs."). Third, an additional item was added at the end of the survey, asking participants how attentive they were when filling out the questionnaire $(1=$ completely attentive, $2=$ moderately attentive, $3=$ not attentive at all).

\section{Procedure}

The study was approved by the Ethics Review Committee Psychology and Neuroscience (ERCPN) of Maastricht University (Ethical Application Ref: RP2027_2019_16). Questionnaires and DCV were assessed via an online survey constructed using Qualtrics ResearchCore ${ }^{\mathrm{TM}}$. Participants were invited at the university to participate in this study. Once seated, participants were welcomed by a researcher and received an information letter and signed a declaration of consent. Next, participants started the online assessment in a university room. Particularly, participants were provided with the instructions of the DCV method and one non-related example on how the DCV should be completed. After the instructions, participants were provided with one of two DCV item sets. Each DCV item set contained all items, but differed in the order in which the constructs had to be filled out (two random orders were drawn in advance which remained consistent throughout a person's assessment). The order in which the $35 \mathrm{DCV}$ items (including 3 IMC items) were presented was random for each participant. After participants completed these DCV items, they provided demographic information, answered the additional question to detect careless responding, and filled out the questions assessing their physical and mental health (PROMIS ${ }^{\circledR}$ Profile - v2.1 - PROMIS-29). Finally, to reduce careless responding, each participant was forced to spend at least 30 seconds on each question to avoid quick and random answers. After finishing the survey, participants received an oral debriefing about the purpose of the study. The online assessment lasted on average 45.86 minutes ( $\mathrm{SD}=$ 17.93 minutes). Participants received course credits for participation in the study.

\section{Analyses}

Data collected with the DCV method were analyzed using Bayesian hierarchical models (JAGS version 4.3.0) in R version 3.6.0 (R Core Team, 2019). This methodology allows to perform analyses at measure as well as at item level, while ensuring that estimates do not fall 
239

240

241

242

243

244

245

246

247

248

249

250

251

252

253

254

255

256

257

258

259

260

261

262

263

264

265

266

267

268

269

270

271

272

273

274

275

276

277

278

outside the actual response range [ -10 to +10$]$ (see also Crombez et al., 2020). In the models a different mu parameter was estimated for each construct or measure, depending on the research question (see below). In addition, a random effect for subject and item was added. All parameters received vague priors (normal distributions with a very large standard deviation; see Crombez et al., 2020). The dependent variable was the DCV outcome score (ranging from -10 to +10 ). The mu parameters come from a truncated normal distribution $[-10,10]$ so the credibility intervals only contain sensible values. To generate the posterior samples, we used 4 chains with 20000 iterations each, 5000 being discarded as burn in. Traceplots and Rhat values of 1 indicated that all the chains for the mu parameters reached convergence. The actual analyses were performed in three steps.

First, we investigated whether the items of the TAS-20, PROMIS-A, PROMIS-D, and SHAI questionnaires, assessing 'alexithymia', 'anxiety', 'depression', and 'health anxiety', were indeed most relevant for measuring their respective construct. Separate analyses were run for each measure. A Bayesian hierarchical model was fitted with construct as a fixed effect and subject and item as random effects.

Second, we examined whether the items of the TAS-20 subscales were most relevant for measuring 'alexithymia', compared to 'anxiety', 'depression', and 'health anxiety'. Separate analyses were run for each subscale. A Bayesian hierarchical model was fitted with measure as a fixed effect and subject and item as random effect. Additionally, we investigated whether the items from the TAS-20 subscales, assessing 'difficulty identifying feelings', 'difficulty describing feelings', and 'externally-oriented thinking', were most relevant for measuring the intended key features of alexithymia, i.e., 'difficulty identifying feelings', 'difficulty describing feelings', 'externally-oriented thinking', and 'limited imaginal capacity'.

Finally, a separate Bayesian hierarchical model was fitted for each single item of the TAS-20. The models included construct as a fixed effect and subject as a random effect. For all models described above, significance was evaluated at the 5\% significance level (two-sided). Estimated mu parameters $(\hat{\mu})$ and their associated $95 \%$ credibility intervals $(\mathrm{CI})$ are reported.

\section{Results}

\section{Participants}

Data from 81 participants (63 females) were collected. After application of the manipulation checks (see section Detection of careless responding), data of 12 participants was removed from further analyses. More specifically, six participants failed to respond correctly to at least one of the IMC items, five participants provided unreliable data (i.e., at least one item was scored as -10 or +10 for all constructs), and one participant indicated that he/she was not attentive at all while completing the questionnaire. The final sample contained 69 participants (mean age of 21.07 years, $S D=1.44 ; 12$ males). Most participants reported their ethnicity as Caucasian $(n=61)$. The large majority of participants $(86 \%)$ reported to be mentally and physically healthy, $9 \%$ reported to be mentally troubled, $1 \%$ reported to be physically troubled, and $4 \%$ reported to be mentally and physically troubled. For the PROMIS, T-scores were 54.49 
$279(S D=4.95 ;$ range $=35.60-57.00)$ for physical function, $54.22(S D=8.29$; range $=40.30-77.90)$

280 for anxiety, $50.36(S D=8.26$; range $=41.00-79.40)$ for depression, $52.89(S D=9.10$; range $=$

$28133.70-75.80)$ for fatigue, $48.22(S D=7.92$; range $=32.00-68.80)$ for sleep disturbance, 53.75

$282(S D=7.41$; range $=31.80-64.20)$ for ability to participate in social roles and activities, $46.62(S D$

$283=7.47$; range $=41.60-75.60)$ for pain interference. A mean score of $1.65(S D=1.92$; range $=0$ -

284 8) was observed for pain intensity.

285 Content validity of TAS-20 questionnaire and questionnaires of related constructs

286 TAS-20 questionnaire

287 The items of the TAS-20 questionnaire scored significantly higher on 'alexithymia' $(\hat{\mu}=$

$2881.12,95 \%$ CI [-0.05 to 2.28]), compared to 'anxiety' ( $\hat{\mu}=-3.48,95 \%$ CI [-4.65 to -2.31$] ; \Delta=$

$2894.60,95 \%$ CI [4.16 to 5.05]), 'depression' ( $\hat{\mu}=-3.85,95 \%$ CI [-5.03 to -2.68$]$; $\Delta=4.98,95 \%$ CI

290 [4.53 to 5.42]), and 'health anxiety' $(\hat{\mu}=-5.13,95 \%$ CI [-6.31 to -3.97$] ; \Delta=6.25,95 \%$ CI [5.80

291 to 6.69]). It should be noted though that the score for 'alexithymia' was not significantly

292 different from zero. Findings are displayed in Figure 1.

293 PROMIS-A questionnaire

294

295

The items of the PROMIS-A questionnaire scored significantly higher on 'anxiety' $(\hat{\mu}=$ 7.93, 95\% CI [6.37 to 9.28]), compared to 'alexithymia' ( $\hat{\mu}=-5.80,95 \%$ CI [-7.39 to -4.45$] ; \Delta=$

296 $13.74,95 \%$ CI [12.85 to 14.62$])$, 'depression' ( $\hat{\mu}=1.73,95 \%$ CI [0.14 to 3.09]; $\Delta=6.20,95 \%$ CI

297 [5.32 to 7.10$]$ ), and 'health anxiety' ( $\hat{\mu}=3.86,95 \%$ CI [2.26 to 5.21$] ; \Delta=4.08,95 \%$ CI [3.19 to 298 4.97]; Figure 1).

299

PROMIS-D questionnaire

300

Similar results were found for the PROMIS-D, showing that the items of the PROMIS-D

301

302

303

304

305

306

307

308

309

310

311

312

313

314

315

316

317

318 scored significantly higher on 'depression' ( $\hat{\mu}=8.40,95 \%$ CI [6.31 to 9.77]), compared to 'alexithymia' ( $\hat{\mu}=-5.90,95 \%$ CI [-8.00 to -4.46$] ; \Delta=14.30,95 \%$ CI [13.45 to 15.16$]$ ), 'anxiety' $(\hat{\mu}=2.33,95 \%$ CI $[0.24$ to 3.78$] ; \Delta=6.07,95 \%$ CI [5.20 to 6.93]), and 'health anxiety' ( $\hat{\mu}=-2.15$ , $95 \%$ CI [-4.25 to -0.70$] ; \Delta=10.55,95 \%$ CI [9.70 to 11.41 ; Figure 1 ).

\section{SHAI questionnaire}

The items of the SHAI questionnaire scored significantly higher on 'health anxiety' $(\hat{\mu}=$ $8.95,95 \%$ CI [7.98 to 9.81]), compared to 'alexithymia' ( $\hat{\mu}=-6.69,95 \%$ CI [-7.66 to -5.78$] ; \Delta=$ $15.64,95 \%$ CI [14.84 to 16.42$])$, 'anxiety' ( $\hat{\mu}=4.54,95 \%$ CI [3.56 to 5.46$] ; \Delta=-6.06,95 \%$ CI [3.62 to 5.21]), and 'depression' ( $\hat{\mu}=-0.63,95 \%$ CI [ -1.61 to 0.29$] ; \Delta=9.58,95 \%$ CI [ 8.78 to 10.37]; Figure 1).

\section{Content validity of the TAS-20 subscales}

\section{Difficulty identifying feelings subscale}

Analyses indicated that the items of the 'difficulty identifying feelings' subscale scored significantly higher on 'alexithymia' ( $\hat{\mu}=3.58,95 \%$ CI [2.10 to 5.12]), compared to 'anxiety' ( $\hat{\mu}$ $=-1.56,95 \%$ CI [ -3.05 to -0.02$] ; \Delta=5.14,95 \%$ CI [4.31 to 5.97]), 'depression' ( $\hat{\mu}=-2.84,95 \%$ CI [-4.32 to -1.30$] ; \Delta=6.42,95 \%$ CI [5.60 to 7.25]), and 'health anxiety' $(\hat{\mu}=-2.16,95 \%$ CI [3.65 to -0.62$] ; \Delta=5.75,95 \%$ CI [4.92 to 6.57$]$; Figure $2 \mathrm{~A}$ ). 
319

320

321

322

323

324

325

326

327

328

329

330

331

332

333

334

335

336

337

338

339

340

341

342

343

344

345

346

347

348

349

350

351

352

353

354

355

356

357

358

Furthermore, the items of the 'difficulty identifying feelings' subscale scored highest on 'difficulty identifying feelings' ( $\hat{\mu}=5.62,95 \%$ CI [3.06 to 7.75]). Yet, compared to 'difficulty describing feelings', the difference was not significant $(\hat{\mu}=2.70,95 \% \mathrm{CI}[0.15$ to 4.81$] ; \Delta=2.92$, $95 \%$ CI [2.18 to 3.65]). Furthermore, items of the 'difficulty identifying feelings' subscale scored significantly higher compared to 'externally-oriented thinking' ( $\hat{\mu}=-4.53,95 \%$ CI [-7.06 to -2.42$] ; \Delta=10.15,95 \% \mathrm{CI}$ [9.42 to 10.88$]$ ), and 'limited imaginal capacity' ( $\hat{\mu}=-3.59,95 \% \mathrm{CI}$ [-6.15 to -1.48$] ; \Delta=9.21,95 \%$ CI [8.48 to 9.94$]$; Figure $2 \mathrm{~B})$.

\section{Difficulty describing feelings subscale}

Analyses showed that the items of the 'difficulty describing feelings' subscale scored significantly higher on 'alexithymia' ( $\hat{\mu}=4.42,95 \%$ CI [2.42 to 6.27]), compared to 'anxiety' $(\hat{\mu}$ $=-4.08,95 \%$ CI [-6.07 to -2.23$]$; $\Delta=8.49,95 \%$ CI [ 7.66 to 9.33]), 'depression' ( $\hat{\mu}=-3.57,95 \%$ CI [-5.56 to -1.72]; $\Delta=7.99,95 \%$ CI [7.15 to 8.83]), and 'health anxiety' ( $\hat{\mu}=-6.84,95 \%$ CI [8.83 to -4.97 ]; $\Delta=11.25,95 \% \mathrm{CI}$ [10.41 to 12.09 ]; Figure $2 \mathrm{~A}$ ).

Furthermore, the items of the 'difficulty describing feelings' subscale scored significantly higher on 'difficulty describing feelings' ( $\hat{\mu}=6.72,95 \%$ CI [4.56 to 8.65]), compared to 'difficulty identifying feelings' ( $\hat{\mu}=1.97,95 \%$ CI [-0.19 to 3.91$] ; \Delta=4.74,95 \%$ CI [3.82 to 5.67]), 'externally-oriented thinking' ( $\hat{\mu}=-5.06,95 \%$ CI [-7.20 to -3.14$] ; \Delta=11.78,95 \%$ CI [10.86 to 12.70$])$, and 'limited imaginal capacity' ( $\hat{\mu}=-3.45,95 \%$ CI $[-5.59$ to -1.53$] ; \Delta=10.16$, 95\% CI [9.24 to 11.08$]$ ) (see Figure 2B).

\section{Externally-oriented thinking subscale}

The items of the 'externally-oriented thinking' subscale scored highest on 'alexithymia' ( $\hat{\mu}=-3.05,95 \%$ CI [ -4.13 to -1.93$])$. Yet, there is no significant difference compared to 'anxiety' ( $\hat{\mu}=-4.76,95 \%$ CI [-5.84 to -3.63$])(\Delta=1.71,95 \%$ CI [1.11 to 2.30$])$, and 'depression' ( $\hat{\mu}=-4.89$, 95\% CI [-5.97 to -3.76]) ( $\Delta=1.83,95 \% \mathrm{CI}[1.24$ to 2.43$])$. 'Alexithymia' scored significantly higher compared to 'health anxiety' ( $\hat{\mu}=-6.62,95 \%$ CI [-7.70 to -5.49$])(\Delta=3.57,95 \%$ CI [2.97 to 4.16]). However, note that $\hat{\mu}$ was negative for all constructs, indicating that the items of the 'externally-oriented thinking' subscale were not endorsed to measure 'alexithymia', nor 'anxiety', 'depression', or 'health anxiety' (Figure 2A).

Furthermore, the items of the 'externally-oriented thinking' subscale scored highest on 'external oriented thinking'. ( $\hat{\mu}=-2.16,95 \%$ CI [-3.69 to -0.62$]$ ). Yet, no significant difference was found compared to 'difficulty describing feelings' ( $\hat{\mu}=-3.26,95 \%$ CI [-4.79 to -1.73$] ; \Delta=$ $1.10,95 \% \mathrm{CI}$ [0.37 to 1.82$])$, 'difficulty identifying feelings' ( $\hat{\mu}=-2.57,95 \% \mathrm{CI}[-4.09$ to -1.02$]$; $\Delta=0.41,95 \%$ CI [-0.31 to 1.14$]$ ), and 'limited imaginal capacity' ( $\hat{\mu}=-3.28,95 \%$ CI [-4.82 to $1.73] ; \Delta=1.13,95 \% \mathrm{CI}[0.41$ to 1.85$])$. Also here, note that $\hat{\mu}$ was negative for all constructs, indicating that the items of the 'externally-oriented thinking' subscale were not endorsed to measure 'externally-oriented thinking', nor 'difficulty describing feelings', 'difficulty identifying feelings', or 'limited imaginal capacity' (Figure 2B).

Figure 2 about here

Content validity of TAS-20 items

Difficulty identifying feelings items

Peer) reviewing PDF | (2020:12:56703:1:0:NEW 16 May 2021) 
359

360

361

362

363

364

365

366

367

368

369

370

371

372

373

374

375

376

377

378

379

380

381

382

383

384

385

386

387

388

389

390

391

392

393

394

395

396

397

398

The 'difficulty identifying feelings' subscale of the TAS-20 contains 7 items (items 1, 3, $6,7,9,13$, and 14). Results indicated that for all items, except item $3, \hat{\mu}$ was positive and the confidence interval did not include 0 , indicating that these items were endorsed to measure 'difficulty identifying feelings' (Figure 1 in S1 file). Furthermore, item $6(\hat{\mu}=8.36,95 \%$ CI [6.99 to 9.66$]$ ) and item 9 ( $\hat{\mu}=8.17,95 \%$ CI [6.73 to 9.57]) scored significantly higher on 'difficulty identifying feelings' than on all other constructs. For item 1, 13 and 14, the score on 'difficulty identifying feelings' (item 1: $\hat{\mu}=7.078,95 \%$ CI [5.68 to 8.49]; item 13: $\hat{\mu}=7.60,95 \%$ CI [6.04 to 9.13]; item 14: $\hat{\mu}=5.66,95 \%$ CI [4.16 to 7.16]) was significantly higher for all constructs, except for 'difficulty describing feelings' (item 1: $\hat{\mu}=4.41,95 \%$ CI [3.01 to 5.82]; item 13: $\hat{\mu}=5.90$, 95\% CI [4.33 to 7.46]; item 14: $\hat{\mu}=3.32,95 \%$ CI [1.83 to 4.81]). For item 3, results indicated a significantly higher score on 'health anxiety' ( $\hat{\mu}=6.54,95 \%$ CI [5.02 to 8.04]) compared to all other constructs, including 'difficulty identifying feelings' $(\hat{\mu}=-1.74,95 \%$ CI [-3.24 to -0.25$])$. A significant positive score was also found for 'anxiety' $(\hat{\mu}=1.8895 \%$ CI $[0.39 ; 3.38]$. Finally, item 7 scored significantly higher on 'difficulty identifying feelings' ( $\hat{\mu}=4.45,95 \%$ CI [2.88 to 6.01]), than on all other constructs, except for 'health anxiety' ( $\hat{\mu}=2.59,95 \%$ CI [1.03 to 4.15]; $\Delta=1.85$, $95 \%$ CI [ -0.20 to 3.92$]$ ). A more detailed description and tabulation of the results is also provided in supplementary information (Table 5 in S1 file).

\section{Difficulty describing feelings items}

The 'difficulty describing feelings' subscale of the TAS-20 contains 5 items (items 2, 4, 11,12 , and 17). Results indicated that for all items, $\hat{\mu}$ was positive and the confidence interval did not include 0 , indicating that these items were endorsed to measure 'difficulty describing feelings' (Figure 2 and Table 5 in S1 file). In addition, item 2 ( $\hat{\mu}=8.42,95 \%$ CI [7.04 to 9.70]), item $11(\hat{\mu}=8.04,95 \%$ CI [6.68 to 9.37]), item $12(\hat{\mu}=6.49,95 \%$ CI [4.99 to 7.99]) and item 17 ( $\hat{\mu}=6.72,95 \%$ CI [5.22 to 8.24]) scored significantly higher on 'difficulty describing feelings' than on all other constructs. For item 4 , the score on 'difficulty describing feelings' $(\hat{\mu}=4.29$, 95\% CI [2.89 to 5.69]) was significantly higher than for all other constructs, except for 'difficulty identifying feelings' ( $\hat{\mu}=2.32,95 \%$ CI [0.92 to 3.75$] ; \Delta=1.97,95 \%$ CI [0.14 to 3.80$]$ ). Externally-oriented thinking items

The 'externally-oriented thinking' subscale of the TAS-20 contains 8 items (items 5, 8, $10,15,16,18,19$, and 20). Results indicated that for item 8 and item $20, \hat{\mu}$ was positive and the confidence interval did not include 0 , indicating that these items were endorsed to measure 'externally-oriented thinking' (Figure 3 and Table 5 in S1 file). For both items, the score on 'externally-oriented thinking' (item $8: \hat{\mu}=1.48,95 \%$ CI [0.06 to 2.91]; item 20: $\hat{\mu}=1.45,95 \%$ CI [0.09 to 2.79]) was significantly higher than on all other constructs. For item 15 , results indicated that $\hat{\mu}$ was positive but the confidence interval did include 0 , indicating that this item was not endorsed to measure 'externally-oriented thinking'. Item 15 was however endorsed to measure 'difficulty describing feelings' ( $\hat{\mu}=2.38,95 \%$ CI [0.88 to 3.86]). For all other items (item 5: $\hat{\mu}=$ 5.01, 95\% CI [-6.22 to -3.79 ]; item 10: $\hat{\mu}=-3.86,95 \%$ CI [-5.28 to -2.44$]$; item 16: $\hat{\mu}=-1.1522$, 95\% CI [-2.66 to 0.36]; item 18: $\hat{\mu}=-5.4109,95 \%$ CI [-6.75 to -4.08$]$; item 19: $\hat{\mu}=-4.92,95 \%$ CI [-6.19 to -3.64$]), \hat{\mu}$ was negative, indicating that these items were not endorsed to measure 
399

400

401

402

403

404

405

406

407

408

409

410

411

412

413

414

415

416

417

418

419

420

421

422

423

424

425

426

427

428

429

430

431

432

433

434

435

436

437

438

'externally-oriented thinking'. For these items, also all other constructs were non-significant or significantly negative, showing that the items were not endorsed to measure any of these constructs either.

\section{Discussion}

The present study investigated the content and discriminant content validity of the TAS-20, a widely used self-report measure of alexithymia (Bagby et al., 2020). Using the Discriminant Content Validity method (DCV; Johnston et al., 2014), participants rated the extent to which each TAS-20 item was relevant for measuring 'alexithymia' and its key features (content validity), or related constructs, i.e., 'anxiety', 'depression', and 'health anxiety' (discriminant content validity). The results can be readily summarized. First, results showed that participants did not endorse the TAS-20 as measuring 'alexithymia', whereas the PROMIS-A, PROMIS-D, and SHAI did distinctively measure their intended construct. Second, the subscales 'difficulty identifying feelings' and 'difficulty describing feelings' measure 'alexithymia'. This was not the case for the 'externally-oriented thinking' subscale. Additionally, results indicated that the 'difficulty describing feelings' subscale distinctively assessed its intended construct. This was not the case for the 'difficulty identifying feelings' subscale, which measures both 'difficulty identifying feelings' and 'difficulty describing feelings'. Furthermore, the 'externally-oriented thinking' subscale assessed none of the included constructs. Finally, results showed that eight items distinctively measured their intended construct, and four items measured both the 'difficulty identifying feelings' and the 'difficulty describing feelings' constructs. Two items assessed (health) anxiety equally well or even better, and none of the items (except item 3) showed content overlap with 'anxiety' or 'depression'.

To our knowledge, this study is the first to empirically investigate the content of the TAS20. Until now content validity has been largely overlooked at the expense of other forms of validity, such as construct (i.e., convergent and discriminant validity) and criterion validity (i.e., predictive, concurrent, and retrospective validity) (e.g., Lumley et al., 2007; Parker, Taylor, \& Bagby, 2003; Bagby et al., 2020). This is surprising as content validity is a fundamental property of any measure of any theoretical construct (Haynes, 1995) and key in theory testing, intervention design, and practical applications (Dixon \& Johnston, 2019; Van Ryckeghem, 2020).

The results of the current study call for reflection. First, overall, the TAS-20 was not considered relevant for measuring 'alexithymia'. Furthermore, the 'difficulty identifying feelings' subscale assessed both the 'difficulty identifying feelings' and the 'difficulty describing feelings' constructs, and the 'externally-oriented thinking' scale was not identified as measuring 'externally-oriented thinking', nor 'alexithymia'. These findings put a threat on the interpretation of earlier and future studies using the TAS-20 as findings are potentially flawed due to a lack of content validity. Nonetheless, the finding that only the 'difficulty identifying feelings' subscale and the 'difficulty describing feelings' subscale were content valid for 'alexithymia' is not surprising. Sifneos' (1973, p. 256) stated that "for lack of a better term", he proposed the term 
"alexithymic" (from Greek stems a = lack, lexis = word, and thymos = mood or emotion) to denote "the most striking characteristic", namely the inability of these patients to find appropriate words to describe their feelings (Nemiah \& Sifneos, 1970). Although the literal meaning of the term alexithymia - 'without words for feelings' - refers to this particular characteristic (Apfel \& Sifneos, 1979), Sifneos made repeatedly clear that the term 'alexithymia' is the name of a construct that encompasses multiple characteristics (e.g., Nemiah et al., 1976; Sifneos, 1994, 1996). Therefore, to define the alexithymia construct in our study, we chose not to use the literal meaning but instead turn to the definition that is used in scientific literature (Taylor et al., 2016) and understood in lay terms (online Oxford Living Dictionaries for English) - 'The inability to recognize one's own emotions and to express them, especially in words.' Although this definition has a broad scope, its focus is on only two out of the four key features, namely the 'difficulty identifying feelings' feature referring to the inability to recognize and the 'difficulty describing feelings' feature referring to the inability to express. In line with this reasoning, we see a plausible explanation for the finding that the 'externally-oriented thinking' subscale was not identified to measure the alexithymia construct. However, by including each of the definitions of the key features, we expected that the 'externally-oriented thinking' subscale would be identified as a measure of the externally-oriented thinking construct, and potentially as an indirect measure of the limited imaginal capacity construct. This was not the case. Analyses of the individual externally-oriented thinking items corroborated this finding. Only two items of this scale were perceived as measuring externally-oriented thinking and none as measuring limited imaginal capacity. Closer inspection of these results showed that four of the items that did not perform well, were reverse-scored items. However, this is not a full explanation because the two other items were regularly keyed. This observation is in line with the findings of Preece et al. (2018), who performed confirmatory factor analysis and showed the presence of a reversescored item method factor, but also poor factor loadings for the regular-keyed items. Our findings are important as they signal the need of revising the items designed to measure externally-oriented thinking so that they represent their intended construct more accurately. Furthermore, our findings contradict Bagby and colleagues' (1994a, 1994b) assumption on the representation of the limited imaginal capacity feature in externally-oriented thinking items. The present results indicate that caution is warranted in using the TAS-20 in its entirety: only two out of the four key features of the alexithymia construct are represented in the item pool.

We also observed that multiple difficulty identifying feelings items had higher scores on the 'difficulty identifying feelings' construct than on the 'difficulty describing feelings' construct. Furthermore, one difficulty describing feelings item had higher scores on the 'difficulty describing feelings' construct than on the 'difficulty identifying feelings' construct. These findings are in line with the results of various studies, revealing that difficulty identifying feelings items and difficulty describing feelings items are closely related, and subscale scores often correlate highly (e.g., $r=.43-.80$; Kooiman et al., 2002). Current findings suggest that content overlap between both subscales may (at least partly) be the basis of observed correlations. Together with the fact that some studies showed that the items of these subscales 
479

480

481

482

483

484

485

486

487

488

489

490

491

492

493

494

495

496

497

498

499

500

501

502

503

504

505

506

507

508

509

510

511

512

513

514

515

516

517

518

merge into one single factor (e.g., Erni, Lötscher, \& Modestin, 1997; Loas, Otmani, Verrier, Fremaux, \& Marchand, 1996), current findings provide support for the idea that part of the items on these scales probably represent the same aspect of alexithymia (Kooiman et al., 2002, but see Gignac et al., 2007). Future research is needed to examine why the wording and phrasing of some of these items is perceived as measuring both constructs.

Finally, two TAS-20 items of the 'difficulty identifying feelings' scale that are developed to measure the difficulty in differentiating between bodily feelings and emotions showed to measure other constructs. One TAS-20 item was identified to measure 'anxiety' and 'health anxiety' (i.e., "I have physical sensations that even doctors don't understand."), the other item was identified to measure both 'health anxiety' and 'difficulty identifying feelings' (i.e., "I am often puzzled by sensations in my body"). Due to this content overlap, these TAS-20 items may result in a misleading evaluation of patients suffering from medically unexplained symptoms. A total score on the TAS-20 may overestimate the prevalence or severity of alexithymia in patients with medical conditions. To avoid unduly psychologization, caution is warranted in interpreting the TAS-20 overall score as a straightforward measure of alexithymia in these populations. One possibility would be to make a separate scale of these items allowing to check/control for their contribution in the TAS-20 total score. A recent study of Fournier and colleagues (2020) provides a potential starting point. In line, with current findings they found that both item 3 and item 7 form a new latent factor difficulty in interoceptive abilities that is specifically related to health and personality trait outcomes. Alternatively, current findings may indicate that a standalone use of this self-report questionnaire may be insufficient to assess alexithymia. There is a need to take into account the context. Indeed, the use of a multimethod approach including the TAS-20, accompanied by a clinical interview and expert judgement may be required to assess whether elevation of self-reported alexithymia levels can be attributed to different aspects of the presentation of alexithymia, the context of the person, or comorbid medical conditions (see also Bagby et al., 2006; Taylor et al., 2016). Future research is warranted to further scrutinize this topic.

Finally, the TAS-20 items did show discriminant content validity with 'anxiety' (except item 3) and 'depression'. This supports the idea that the TAS-20 is not merely a measure of negative affect (Lumley, 2000; Bagby et al., 2020), indicating that high correlations between alexithymia and anxiety/depression are not due to content overlap between scales of both constructs. Yet, it remains possible that the high correlations between alexithymia and negative affect are due to the particular formulation of a substantial part of the TAS-20 items. Indeed, a substantial number of items of the 'difficulty identifying feelings' subscale and the 'difficulty describing feelings' subscale are negatively phrased (e.g., "I find it hard to describe how I feel about people"). It is known that people high in negative affectivity tend to manifest a general tendency towards a self-effacing response style or self-criticism, thus, tend to report negative things about themselves on self-report questionnaires generally (Lumley et al., 2000).

This study has some limitations. First, healthy lay people, and no experts or patients were involved. The nature of the discriminant validity method is designed to allow lay people without 
519 scientific background (and thus knowledge biases) to judge whether items assess a certain 520 construct (see also Crombez et al., 2020). However, no agreement exists whether experts should

521 be used who are familiar with the theoretical constructs, or whether non-biased lay people should

522 be used who are the putative respondents of the measure (Dixon \& Johnston, 2019). Second, 523 judges were mainly female, which precluded the examination of gender effects. Third, the DCV 524 method provides a quantitative analysis of content validity. Other methods are possible, and may 525 provide insight in how participants mentally process and respond to items. One promising 526 procedure to provide a qualitative analysis of content validity is cognitive interviewing (Willis, 527 2015). Fourth, the statistical analyses differed from the analyses performed in typical research 528 with the DCV method (Johnston et al., 2014, but see Crombez et al., 2020). These studies 529 generally involve a low number of participants (at least 15 participants are recommended) and 530 use primarily one-sample or paired t-tests. Hence, results then strongly depend on sample size 531 and statistical power. The analyses in the present research were performed using Bayesian 532 hierarchical models, which has several advantages (see earlier), but also requires a larger number 533 of participants. Therefore, the sample size of current study largely exceeds previous studies 534 resulting in narrow confidence intervals. Fifth, we have only included the TAS-20. Other 535 measures exist such as the Bermond Vorst Alexithymia Questionnaire (BVAQ; Vorst \& 536 Bermond, 2001), the Psychological Treatment Inventory-Alexithymia Scale (PTI-AS; Gori et al., 537 2010), and the Perth Alexithymia Questionnaire (PAQ; Preece, Becerra, Robinson, Dandy, \& 538 Allan, 2018). Although promising results on the psychometric properties of the latter measures 539 have been reported (see de Vroege et al. 2018; Bagby et al., 2009; Vorst \& Bermond, 2001), 540 future research is needed to corroborate the (discriminant) content validity of these

541 questionnaires as well.

542

543

544

545

546

547

\section{Conclusions}

The TAS-20, currently the most utilized instrument to assess alexithymia and its key features is found to be only partially content valid. Particularly, current findings indicate problems with the content validity of the TAS-20 questionnaire, and some of its subscales do not measure the intended key features. Indeed, only the subscales 'difficulty identifying feelings' and 'difficulty describing feelings' represented 'alexithymia' and their intended construct. This was not the case for the 'externally-oriented thinking' subscale, which assessed none of the alexithymia key features or related constructs. Finally, some items of the TAS-20 are contaminated with content measuring (health) anxiety. Due to described problems with (discriminant) content validity, revision of the TAS-20 is recommended to adequately assess (all key features of) alexithymia. Furthermore, caution is warranted when assessing the TAS-20 in people suffering from medical conditions.

\section{Acknowledgements}

We thank Clara Coen, Elena Dhondt, Thea Herfs, Arawa Kolossa, Lynn Pasch, Glenn Proctor, Marie Santillo, and Dyonne Vrouenraets for their help in the data collection. 


\section{References}

561

562

563

564

565

566

567

568

569

570

571

572

573

574

575

576

577

578

579

580

581

582

583

584

585

586

587

588

589

590

591

592

593

594

595

596

597

598

Bagby, R. M., Parker, J. D. A., \& Taylor, G. J. (1994a). The twenty-item Toronto Alexithymia scale-I. Item selection and cross-validation of the factor structure. Journal of Psychosomatic Research, 38, 23-32. https://doi.org/10.1016/0022-3999(94)90005-1

Bagby, R. M., Parker, J., \& Taylor, G. J. (2020). Twenty-five years with the 20 -item Toronto Alexithymia Scale. Journal of psychosomatic research, 131, 109940. Advance online publication. https://doi.org/10.1016/j.jpsychores.2020.109940

Bagby, R. M., Taylor, G. J., \& Parker, J. D. A. (1994b). The twenty-item Toronto Alexithymia scale-II. Convergent, discriminant, and concurrent validity. Journal of Psychosomatic Research, 38, 33-40. https://doi.org/10.1016/0022-3999(94)90006-X

Bagby, R. M., Taylor, G. J., Parker, J. D., \& Dickens, S. E. (2006). The development of the Toronto Structured Interview for Alexithymia: item selection, factor structure, reliability and concurrent validity. Psychotherapy and Psychosomatics, 75(1), 25-39. https://doi.org/10.1159/000089224

Bagby, R. M., Quilty, L. C., Taylor, G. J., Grabe, H. J., Luminet, O., Verissimo, R., De Grootte, I., \& Vanheule, S. (2009). Are there subtypes of alexithymia? Personality and Individual Differences, 47(5), 413-418. https://doi.org/10.1016/j.paid.2009.04.012

Barsky, A. J. (2001). Somatosensory, Amplification and hypochondriasis. In V. Starcevic \& D. R. Lipsitt (Eds.), Hypochondriasis: Modern perspectives on an ancient malady (pp. 223248). NewYork: Oxford University Press.

Bermond, B., Oosterveld, P., \& Vorst, H. C. M. (2015). Measures of alexithymia. In G. J. Boyle, D. H. Saklofske, \& G. Matthews (Eds.), Measures of Personality and Social Psychological Construct (pp. 227-256). London, UK: Academic Press.

Bräutigam, W., \& von Rad, M. (Eds.) (1977). Toward a theory of psychosomatic disorders : alexithymia, pensée opératoire, psychosomatisches phänomen: proceedings of the 11th European Conference on Psychosomatic Research, Heidelberg, September 14-17, 1976. Basel: Karger.

Corcos, M., \& Speranza, M. (2003). Psychopathologie de l'alexithymie. Paris: Dunod.

Crombez, G., De Paepe, A. L., Veirman, E., Eccleston, C., Verleysen, G., \& Van Ryckeghem, D. M. L. (2020). Let's talk about pain catastrophizing measures: an item content analysis. PeerJ. 8:e8643. https://doi.org/10.7717/peerj.8643

De Gucht, V., Fischler, B., \& Heiser, W. (2004). Personality and affect as determinants of medically unexplained symptoms in primary care; a followup study. Journal of Psychosomatic Research, 56, 279-285. https://doi.org/10.1016/S0022-3999(03)00127-2

de Vroege, L., Emons, W., Sijtsma, K., \& van der Feltz-Cornelis, C. M. (2018). Psychometric Properties of the Bermond-Vorst Alexithymia Questionnaire (BVAQ) in the General Population and a Clinical Population. Frontiers in psychiatry, 9, 111. https://doi.org/10.3389/fpsyt.2018.00111

Dixon, D., \& Johnston, M. (2019). Content validity of measures of theoretical constructs in

Peer) reviewing PDF | (2020:12:56703:1:0:NEW 16 May 2021) 
599

600

601

602

603

604

605

606

607

608

609

610

611

612

613

614

615

616

617

618

619

620

621

622

623

624

625

626

627

628

629

630

631

632

633

634

635

636

637

638

health psychology: Discriminant content validity is needed. British Journal of Health Psychology, 24, 477-484. https://doi.org/10.1111/bjhp.12373

Erni, T., Lötscher, K., \& Modestin, J. (1997). Two-factor solution of the 20-Item Toronto Alexithymia Scale confirmed. Psychopathology, 30, 335-340. https://doi.org/10.1159/000285079

Fernandez, K. C., Jazaieri, H., \& Gross, J. J. (2016). Emotion Regulation: A Transdiagnostic Perspective on a New RDoC Domain. Cognitive therapy and research, 40(3), 426-440. https://doi.org/10.1007/s10608-016-9772-2

Fournier, A., Luminet, O., Dambrun, M., Dutheil, F., Pellissier, S., \& Mondillon, L. (2019). Importance of considering interoceptive abilities in alexithymia assessment. PeerJ, 7, e7615. https://doi.org/10.7717/peerj.7615

Frewen, P. A., Pain, C., Dozois, D. J., \& Lanius, R. A. (2006). Alexithymia in PTSD: psychometric and FMRI studies. Annals of the New York Academy of Sciences, 1071, 397400. https://doi.org/10.1196/annals.1364.029

Gignac, G. E., Palmer, B. R., \& Stough, C. (2007). A confirmatory factor analytic investigation of the TAS-20: Corroboration of a Five-factor model and suggestions for improvement. Journal of Personality Assessment, 89, 247-257. https://doi.org/10.1080/00223890701629730

Gori, A., Giannini, M., Palmieri, G., Salvini, R., \& Schuldberg, D. (2012). Assessment of alexithymia: psychometric properties of the Psychological Treatment InventoryAlexithymia Scale (PTI-AS) Psychology, 3, 231-236. https://doi.org/10.4236/psych.2012.33032

Haynes, S., Richard, D., Kubany, E., \& Butcher, J. N. (1995). Content Validity in Psychological Assessment: A Functional Approach to Concepts and Methods. Psychological Assessment, 7, 238-247. https://doi.org/10.1037/1040-3590.7.3.238

Hays, R. D., Marshall, G. N., Wang, E. Y. I., \& Sherbourne, C. D. (1994). Four-year crosslagged associations between physical and mental health in the medical outcomes study. Journal of Consulting and Clinical Psychology, 62, 441-449.

https://doi.org/10.1037//0022-006x.62.3.441

Hays, R. D., Spritzer, K. L., Schalet, B. D., \& Cella, D. (2018). PROMIS®-29 v2.0 Profile Physical and Mental Health Summary Scores. Quality of Life Research, 27, 1885-1891. https://doi.org/10.1007/s11136-018-1842-3

Honkalampi, K., Koivumaa-Honkanen, H., Lehto, S. M., Hintikka, J., Haatainen, K., Rissanen, T., \& Viinamäki, H. (2010). Is alexithymia a risk factor for major depression, personality disorder, or alcohol use disorders? A prospective population-based study. Journal of Psychosomatic Research, 68, 269-273. https://doi.org/10.1016/j.jpsychores.2009.05.010

Johnston, M., Dixon, D., Hart, J., Glidewell, L., Schröder, C., \& Pollard, B. (2014). Discriminant content validity: A quantitative methodology for assessing content of theory-based measures, with illustrative applications. British Journal of Health Psychology, 19, 240-57. https://doi.org/10.1111/bjhp.12095 
639 Kooiman, C. G., Spinhoven, P., \& Trijsburg, R. W. (2002). The assessment of alexithymia. A

640

641

642

643

644

645

646

647

648

649

650

651

652

653

654

655

656

657

658

659

660

661

662

663

664

665

666

667

668

669

670

671

672

673

674

675

676

677

678

critical review of the literature and a psychometric study of the Toronto Alexithymia Scale20. Journal of Psychosomatic Research. 53, 1083-1090. https://doi.org/10.1016/s00223999(02)00348-3

Lane, R. D., Weihs, K. L., Herring, A., Hishaw, A., \& Smith, R. (2015). Affective agnosia: Expansion of the alexithymia construct and a new opportunity to integrate and extend Freud's legacy. Neuroscience \& Biobehavioral Reviews, 55, 594-611. https://doi.org/10.1016/j.neubiorev.2015.06.007

Leising, D., Grande, T., \& Faber, R. (2009). The Toronto Alexithymia Scale (TAS-20): A measure of general psychological distress. Journal of Research in Personality, 43, 707710. https://doi.org/10.1016/j.jrp.2009.03.009

Loas, G., Otmani, O., Verrier, A., Fremaux, D., \& Marchand, M. P. (1996). Factor analysis of the French version of the 20-Item Toronto Alexithymia scale (TAS-20). Psychopathology, 29, 139-144. https://doi.org/10.1016/j.jrp.2009.03.009

Luminet, O., Taylor, G. J., \& Bagby, R. M. (2018). Alexithymia. Advances in Research, Theory, and Clinical Practice. Cambridge University Press: Cambridge, UK.

Lumley, M. A. (2000). Alexithymia and negative emotional conditions. Journal of Psychosomatic Research, 49, 51-54. https://doi.org/10.1016/S0022-3999(00)00161-6

Lumley, M. A., Neely, L. C., \& Burger, A. J. (2007). The Assessment of Alexithymia in Medical Settings: Implications for Understanding and Treating Health Problems. Journal of Personality Assessment, 89, 230-246. https://doi.org/10.1080/00223890701629698

Maclean, P. D. (1949). Psychosomatic disease and the "visceral brain"; recent developments bearing on the Papez theory of emotion. Psychosomatic Medicine, 11, 338-353. https://doi.org/10.1097/00006842-194911000-00003

Marty, P., \& de M'Uzan, M. (1963). La pensée opératoire. Revue française de psychanalyse, 27, 345-355.

Marchesi, C., Ossola, P., Tonna, M., \& De Panfilis, C. (2014). The TAS-20 more likely measures negative affects rather than alexithymia itself in patients with Major Depression, Panic Disorder, Eating Disorders and Substance Use Disorders. Comprehensive Psychiatry, 55, 972-978. https://doi.org/10.1016/j.comppsych.2013.12.008

Meade, A. W., \& Craig, S. B. (2012). Identifying Careless Responses in Survey Data. Psychological Methods, 17, 437-55. https://doi.org/10.1037/a0028085

Nakao, M., Barsky, A. J, Kumano, H., \& Kubaki, T. (2002). Relationship Between Somatosensory Amplification and Alexithymia in a Japanese Psychosomatic Clinic. Psychosomatics, 43, 55-60. https://doi.org/10.1176/appi.psy.43.1.55

Nemiah, J. C., \& Sifneos, P. E. (1970). Psychosomatic illness: A problem in communication. Psychotherapy and Psychosomatics, 18, 154-160. https://doi.org/10.1159/000286074

Nemiah, J. C., Freyberger, H., \& Sifneos, P. E. (1976). Alexithymia: A view of the psychosomatic process. In O. W. Hill (Ed.), Modern trends in psychosomatic research (pp. 430-439). London: Buttersworth.

Peer) reviewing PDF | (2020:12:56703:1:0:NEW 16 May 2021) 
679 Oppenheimer, D. M., Meyvis, T., \& Davidenko, N. (2009). Instructional manipulation checks:

680

681

682

683

684

685

686

687

688

689

690

691

692

693

694

695

696

697

698

699

700

701

702

703

704

705

706

707

708

709

710

711

712

713

714

715

716

717

718

Detecting satisficing to increase statistical power. Journal of Experimental Social

Psychology, 45, 867-72. https://doi.org/10.1016/j.jesp.2009.03.009

Parker, J. D. A., Taylor, G. J., \& Bagby, R. M. (2003). The 20-Item Toronto Alexithymia Scale: III. Reliability and factorial validity in a community population Journal of Psychosomatic Research, 55, 269-275. https://doi.org/10.1016/s0022-3999(02)00578-0

Pilkonis, P. A., Choi, S. W., Reise, S. P., Stover, A. M., Riley, W. T., \& Cella, D. (2011). Item Banks for Measuring Emotional Distress from the Patient-Reported Outcomes Measurement Information System (PROMIS): Depression, Anxiety, and Anger. Assessment, 18, 263-283. https://doi.org/10.1177/1073191111411667

Pecukonis, E. V. (2009). Physical self-efficacy and alexithymia in women with chronic intractable back pain. Pain Management Nursing, 10, 116-123. https://doi.org/10.1016/j.pmn.2008.11.001

Porcelli, P., \& Taylor, G. J. (2018). Alexithymia and physical illness: a psychosomatic approach. In O. Luminet, R. M. Bagby, \& G. J. Taylor (Eds.), Alexithymia: Advances in Research, Theory, and Clinical Practice (pp. 105-126). New York: Cambridge University Press.

Preece, D., Becerra, R., Allan, A., Robinson, K., \& Dandy, J. (2017). Establishing the theoretical components of alexithymia via factor analysis: Introduction and validation of the attentionappraisal model of alexithymia. Personality and Individual Differences, 119, 341-352. https://doi.org/10.1016/j.paid.2017.08.003

Preece, D., Becerra, R., \& Campitelli, G. (2018). Assessing emotional reactivity: Psychometric properties of the Perth Emotional Reactivity Scale and the development of a short form. Journal of Personality Assessment, 101(6), 1-9. https://doi.org/10.1080/00223891.2018.1465430

Rose, A. J., Bayliss, E., Huang, W., Baseman, L, Butcher, E., ..., Orlando Edelen, M. (2018). Evaluating the PROMIS-29 v2.0 for use among older adults with multiple chronic conditions. Quality of Life Research, 27: 2935. https://doi.org/10.1007/s11136-018-1958-5

Ruesch, J. (1948). The infantile personality; the core problem of psychosomatic medicine. Psychosomatic Medicine, 10, 134-144. https://doi/10.1097/00006842-194805000-00002

Salkovskis, P. M., Rimes, K. A., Warwick, H. M. C., \& Clark, D. M. (2002). The Health Anxiety Inventory: Development and validation of scales for the measurement of health anxiety and hypochondriasis. Psychological Medicine, 32, 843-853. https://doi.org/10.1017/s0033291702005822

Sekely, A., Bagby, R. M., \& Porcelli P. (2018). Alexithymia: advances in research, theory, and clinical practice. In O. Luminet, R. M., Bagby, \& G. J. Taylor (eds.). Alexithymia: Advances in Research, Theory, and Clinical Practice (pp. 17-32). Cambridge: Cambridge University Press.

Shahidi, S., Molaie, A., \& Dehghani, M. (2012). Relationship between health anxiety and alexithymia in an Iranian sample. Social and Behavioral Sciences, 46, 591-595. https://doi.org/10.1016/j.sbspro.2012.05.168

Peer) reviewing PDF | (2020:12:56703:1:0:NEW 16 May 2021) 
719 Sifneos, P. E. (1967). Clinical observations on some patients suffering from a variety of

720

721

722

723

724

725

726

727

728

729

730

731

732

733

734

735

736

737

738

739

740

741

742

743

744

745

746

747

748

749

750

751

752

753

754

755

756

757

758

psychosomatic diseases. Acta Medicina Psychosomatica, 7, 1-10.

Sifneos, P. E. (1972). Short-term Psychotherapy and Emotional Crisis. Cambridge: Harvard University Press.

Sifneos, P. E. (1973). The prevalence of 'alexithymic' characteristics in psychosomatic patients. Psychotherapy and Psychosomatics, 22, 255-262. https://doi.org/10.1159/000286529

Sifneos, P. E. (1994). Affect deficit and alexithymia. New Trends in Experimental and Clinical Psychiatry, 10, 193-195.

Sifneos, P. E. (1996). Alexithymia: Past and presence. American Journal of Psychiatry, 153, 137-142. https://doi.org/10.1176/ajp.153.7.137

Sifneos, P. E. (2000). Alexithymia, Clinical Issues, Politics and Crime. Psychotherapy and psychosomatics, 69, 113-116. https://doi.org/10.1159/000012377

Taylor, G. J. (2004). Alexithymia: 25 years of theory and research. In I. Nyklíek, L. Temoshok, \& A. Vingerhoets (Eds.), Emotion Expression and Health: Advances in Theory, Assessment and Clinical Applications (pp. 137-153). New York: Brunner-Routledge.

Taylor, G. J. \& Bagby, R. M. (2000). An overview of the alexithymia construct. In R. Bar-On \& J.D.A. Parker (Eds.), Handbook of Emotional Intelligence (pp. 40-67). San Francisco: Jossey-Bass.

Taylor, G. J. \& Bagby, R. M. (2004) New trends in alexithymia research. Psychotherapy and Psychosomatics, 73, 68-77. https://doi.org/10.1159/000075537

Taylor, G. J., Bagby, R. M., \& Parker, J. D. A. (1992). The Revised Toronto Alexithymia Scale: some reliability, validity, and normative data. Psychotherapy and psychosomatics, 57, 3441. https://doi.org/10.1159/000288571

Taylor, G. J., Bagby, R. M., \& Parker, J. D. A. (1997). Disorders of Affect Regulation: Alexithymia in Medical and Psychiatric Illness. Cambridge: Cambridge University Press.

Taylor, G. J., Bagby, R. M., \& Parker, J. D. A. (2003).The 20-Item Toronto Alexithymia Scale: III. Reliability and factorial validity in a community population. Journal of Psychosomatic Research, 55, 269-275. https://doi.org/10.1016/s0022-3999(02)00578-0

Taylor, G. J., Bagby, R. M., \& Parker, J. D. A. (2016). What's in the name 'alexithymia'? A commentary on "Affective agnosia: Expansion of the alexithymia construct and a new opportunity to integrate and extend Freud's legacy." Neuroscience \& Biobehavioral Reviews, 68, 1006-1020. https://doi.org/10.1016/j.neubiorev.2016.05.025

Taylor, G. J., Parker, J. D., Bagby, R. M, \& Bourke, M. P. (1996). Relationships between alexithymia and psychological characteristics associated with eating disorders. Journal of Psychosomatic Research, 4, 561-568. https://doi.org/10.1016/s0022-3999(96)00224-3

Taylor, G. J., Ryan, D., \& Bagby, R. M. (1985). Toward the development of a new self-report alexithymia scale. Psychotherapy and psychosomatics, 44(4), 191-199. https://doi.org/10.1159/000287912

Todarello, O., La Pesa, M. V., Zaka, S., Martino, V., \& Lattanzio, E. (1989). Alexithymia and breast cancer. Survey of 200 women undergoing mammography. Psychotherapy and

Peer) reviewing PDF | (2020:12:56703:1:0:NEW 16 May 2021) 
759 Psychosomatics; 51, 51-55. https://doi.org/10.1159/000288134

760 Van Ryckeghem, D.M.L. (2021). Accepatnce is not acceptance, but acceptance! European

761 journal of Pain, 25:3-4. https://doi.org/10.1002/ejp.1672

762 Vorst, H. C. M., \& Bermond, B. (2001). Validity and reliability of the Bermond-Vorst

763 Alexithymia Questionnaire. Personality and Individual Differences, 30, 413-434.

764 https://doi.org/10.1016/S0191-8869(00)00033-7

765 Willis, G. B. (2015). Analysis of the cognitive interview in questionnaire design. Oxford, UK:

766 Oxford University Press. 
Figure 1

Estimates and Associated 95\% Credibility Intervals of the Relevance Score for the TAS, $P A, P D$, and SHAl for Alexithymia, Anxiety, Depression, and Health Anxiety.

TAS $=$ Toronto Alexithymia Scale $-20 ;$ PA $=$ PROMIS ${ }^{\circledR}$ Item Bank v1.0-Emotional DistressAnxiety - Short Form 4a; PD = PROMIS ${ }^{\circledR}$ Item Bank v1.0 - Emotional Distress-Depression Short Form 4a; SHAI = Short Health Anxiety Inventory. 


\section{DISCRIMINANT CONTENT VALIDITY TAS-20}

Figure 1

Estimates and Associated 95\% Credibility Intervals of the Relevance Score for the TAS, PA, PD, and SHAI for the Constructs Alexithymia, Anxiety, Depression, and Health Anxiety.
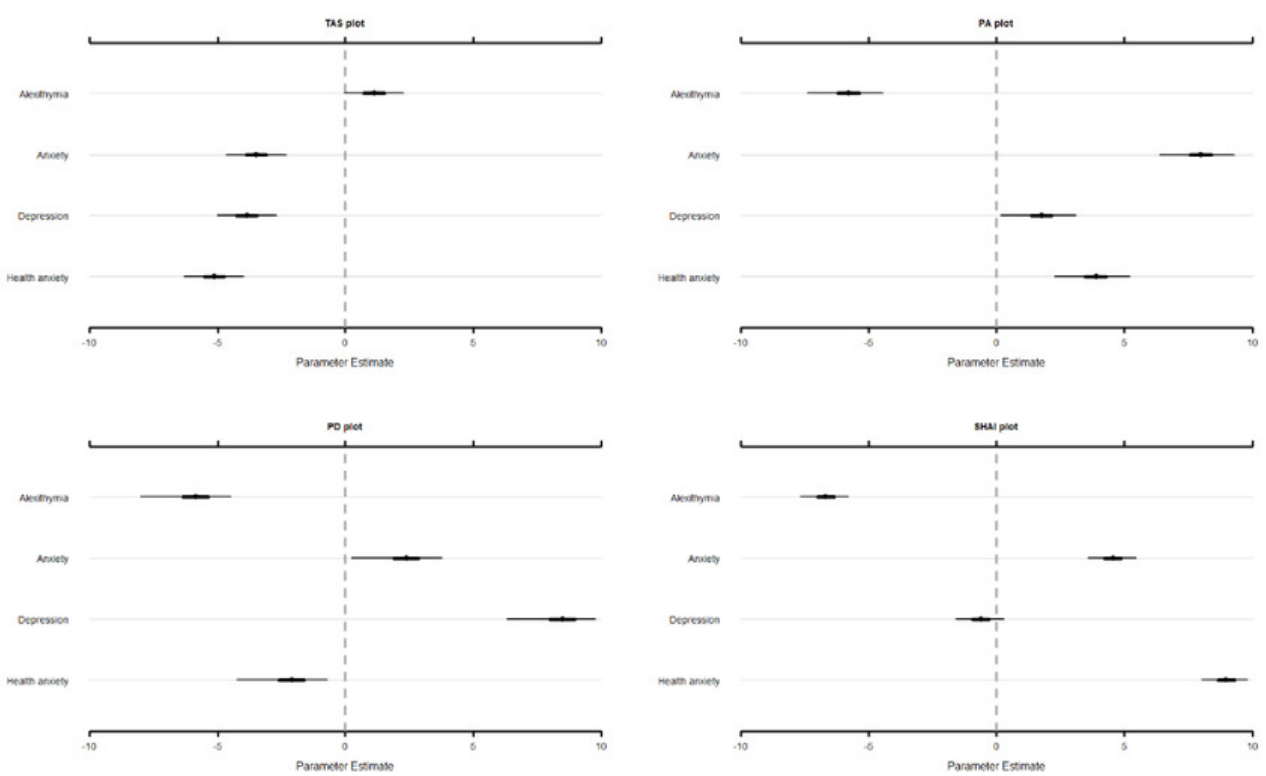

Note. TAS $=$ Toronto Alexithymia Scale $-20 ; \mathrm{PA}=$ PROMIS $^{\circ}$ Item Bank v1.0-Emotional Distress-Anxiety Short Form 4a; PD = PROMIS ${ }^{\circ}$ Item Bank v1.0 - Emotional Distress-Depression - Short Form 4a; SHAI = Short Health Anxiety Inventory. 


\section{Figure 2}

Estimates and Associated 95\% Credibility Intervals of the Relevance Score for each TAS Subscale on the alexithymia key features (A), and Alexithymia, Anxiety, Health Anxiety, and Depression (B).

TAS $=$ Toronto Alexithymia Scale $-20 ;$ PA $=$ PROMIS ${ }^{\oplus}$ Item Bank v1.0-Emotional DistressAnxiety - Short Form 4a; PD = PROMIS ${ }^{\circledR}$ Item Bank v1.0 - Emotional Distress-Depression Short Form 4a; SHAI = Short Health Anxiety Inventory. 


\section{DISCRIMINANT CONTENT VALIDITY TAS-20}

Figure 2

Estimates and Associated 95\% Credibility Intervals of the Relevance Score for each TAS Subscale on the Constructs Difficulty Identifying Feelings, Difficulty Describing Feelings, Externally-Oriented Thinking, and Limited Imaginal Capacity (A), and Alexithymia, Anxiety, Health Anxiety, and Depression (B).

A
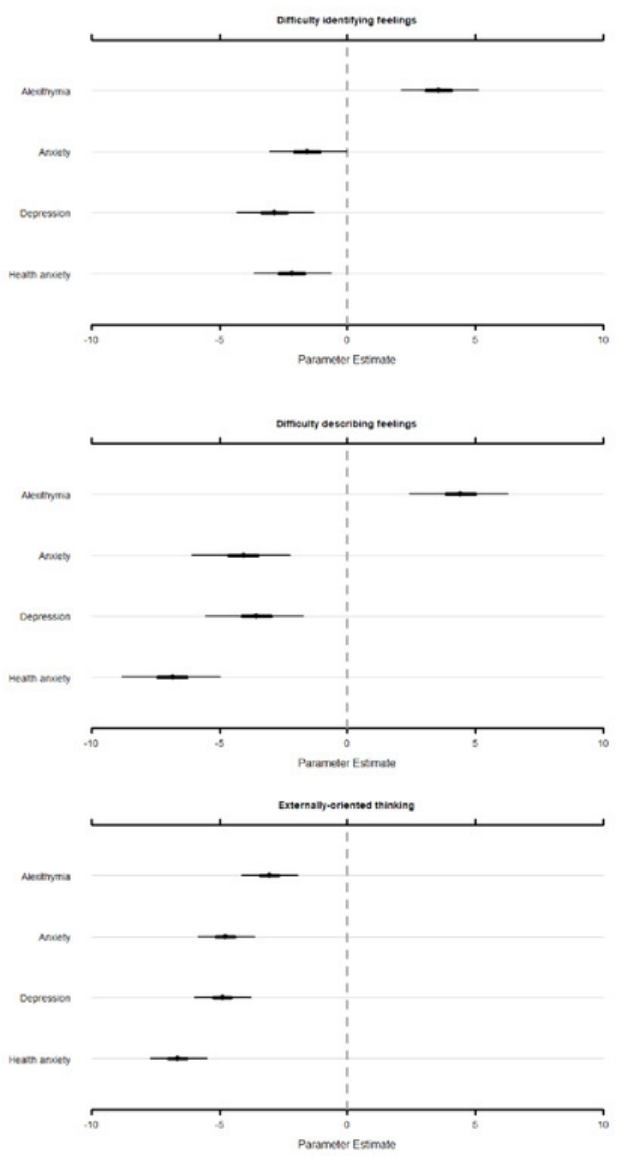

B
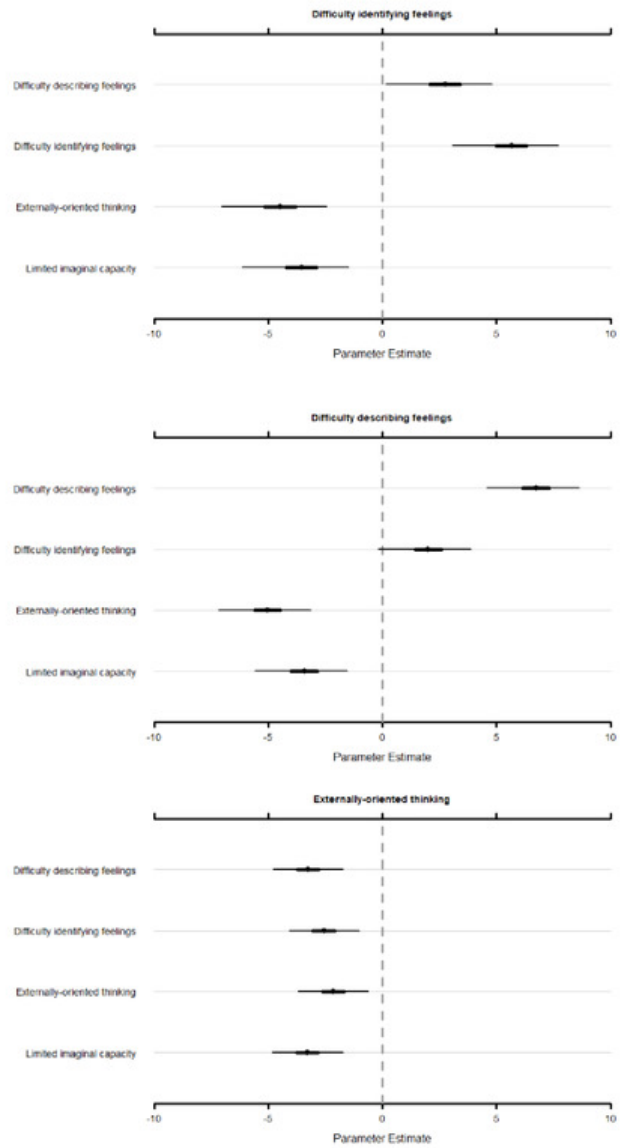

Note. TAS $=$ Toronto Alexithymia Scale - 20; PA = PROMIS Item Bank v1.0-Emotional Distress-Anxiety Short Form 4a; PD = PROMIS ${ }^{\circ}$ Item Bank v1.0 - Emotional Distress-Depression - Short Form 4a; SHAI = Short Health Anxiety Inventory. 